\title{
Evaluación de prácticas de bienestar animal durante el transporte de bovinos para sacrificio
}

\author{
Evaluating animal welfare practice when transporting cattle to \\ slaughter
}

\author{
Marlyn H. Romero, Jorge A. Sánchez y Carolina Gutiérrez
}

Departamento de Salud Animal, Facultad de Ciencias Agropecuarias, Universidad de Caldas. Manizales, Colombia.marlyn.romero@ucaldas.edu.co,jorge.sanchez@ucaldas.edu.co, caroliner2@hotmail.com

Recibido 1 Octubre 2010/Enviado para Modificación 21 Julio 2011/Aceptado 5 Agosto 2011

\section{RESUMEN}

Objetivo Evaluar las prácticas de bienestar animal durante el transporte terrestre de bovinos para el sacrificio.

Métodos Se realizó un estudio transversal analítico en 194 transportadores de bovinosde dos zonas comerciales del occidente colombiano. Se analizaron variables de mográficas, actitudes de los conductores frente a los animales, y las condiciones de los vehículos, de acuerdo con los lineamientos de la legislación sanitaria.

Resultados El transporte de bovinos es realizado por personas con edades comprendidas entre los 31 y 60 años (69 \%), con escasa capacitación en manejo animal $(4,1 \%)$. Los conductores no vigilan las condiciones físicas de los animales durante el viaje, y los manejan con métodos como la picana eléctrica $(46,8 \%)$, torciendo la cola u otros métodos que generan dolor $(24,2 \%)$, por medio de palos $(16,1 \%)$ y lazos $(12,9 \%)$, los cuales no garantizan el bienestar animal. El transporte no es especializado $(79,4 \%)$ y las condiciones de los vehículos cumplen parcialmente con los requerimientos sanitarios.

Conclusiones Se requiere el mejoramiento de la infraestructura de transporte de los bovinos en el área de estudio, la capacitación de los conductores, el fomento de prácticas de bienestar animal en todos los usuarios de la cadena cárnica y el desarrollo de investigación en el área.

Palabras Clave: Ganado, bovino, bienestar del animal (fuente: DeCS, BIREME).

\section{ABSTRACT}

Objective Evaluating animal welfare practices during land transport of cattle to slaughter.

Methods A cross-sectional analytical study was carried out regarding 194 livestock transporters from two cattle-producing areas in western Colombia. Demographic 
variables, drivers' attitudes towards the animals and trucking conditions were analyzed according to sanitary legislation guidelines.

Results The livestock are transported by people aged 31 to 60 (69\%) having little animal management training $(4.1 \%)$. The drivers do not supervise the cattle's physical conditions when they are being transported and handled by methods such as electric prodding (46.8\%), twisting their tails or other pain-inducing methods $(24.2 \%)$, using sticks $(16.1 \%)$ and ropes $(12.9 \%)$ which do not guarantee appropriate animal welfare. Transportation is not specialised $(79.4 \%)$ and the conditions of the vehicles only partially meet sanitary requirements.

Conclusions Cattle transport infrastructure must be improved in the area being studied, as must driver training; animal welfare practice must be promoted in all users of the meat chain and research developed in this particular area.

Key Words: Livestock, cattle, animal well-being (source: MeSH, NLM).

$\mathrm{E}$ n Sur América el transporte de los animales desde los sistemas productivos a las plantas de beneficio se efectúa generalmente por vía terrestre (1). Sin embargo, esta actividad es realizada por personal poco especializado o no capacitado en el transporte de bovinos en pie $(2,3)$. Se ha sugerido que la actitud y la formación de los manejadores de los animales, así como las características de las instalaciones y vehículos, están directamente asociadas con la pérdida de BA $(4,5)$. El BA está íntimamente relacionado con la inocuidad alimentaria, la calidad de la carne y las pérdidas económicas en la cadena cárnica bovina (6). El estrés durante el pre sacrificio (etapas desde el cargue en la finca, hasta la insensibilización y la sangría) favorece la migración de microorganismo intestinales a las masas musculares profundas y posterior crecimiento microbiano; aumenta además, la eliminación de microorganismos entéricos por las excretas (p.e. E. coli O157:H7),y a través de la contaminación cruzada de la carne durante el beneficio, se incrementa el riesgo biológico para el consumidor $(7,8 \%)$. El transporte deficiente puede ocasionar la disminución entre el 1,5 y $9 \%$ del peso vivo, incrementa los riegos de caída, muerte y contusiones, aumento de pérdidas económicas por eliminación de tejido contuso, menor rendimiento en canal y descenso en la categoría de tipificación de las canales, entre otros $(2,6)$. Los animales agotados y estresados proporcionan una carne denominada corte "oscuro" o carne DFD (Dark, firm, dry), que por sus características organolépticas y fisicoquímicas, son poco atractivas para los consumidores, disminuyen su vida útil, favorecen el crecimiento microbiano, limitan las posibilidades de exportación y no son aptas para empacarlas al vacío $(9,10)$.

La Organización Mundial de Sanidad Animal (OIE) es el Ente internacional de referencia en el ámbito del BA, y ha elaborado recomendaciones para que 
los Servicios Veterinarios orienten su implementación durante el transporte y el sacrificio (11). En Colombia mediante la resolución 002341 de 2007 (12), se reglamentaron las buenas prácticas de producción primaria de ganado bovino y bufalino destinado al sacrificio para consumo humano, incluyendo el BA y las prácticas de manejo de los animales durante el transporte. El objetivo del presente trabajo consistió en evaluar las prácticas de BA del transporte terrestre de ganado de dos zonas productoras del occidente colombiano; y evaluar la infraestructura de los vehículos de acuerdo a las directrices de la legislación sanitaria vigente.

\section{MATERIALES Y MÉTODOS}

Recolección de la información

El estudio se realizó durante los períodos agosto-diciembre de 2009 y febreroabril de 2010 en dos frigoríficos categoría 1, localizados en el occidente colombiano en los departamentos de Antioquia y Caldas, que benefician ganado procedente del Magdalena medio y de la costa Colombiana, de los departamentos de Tolima, Caldas, Risaralda, Santander, Valle, Antioquia, Quindío, Boyacá, Caquetá, Cundinamarca, Córdoba, Cesar, entre otros.

Se hizo un estudio transversal analítico, mediante la aplicación de un instrumento estructurado previamente probado mediante una prueba piloto. Se aplicó a 194 conductores, con voluntad de participación mediante el consentimiento informado. Se evaluaron variables demográficas, el manejo de los animales y las condiciones de diseño de los vehículos, de acuerdo a los requerimientos sanitarios establecidos en la Resolución 002341 de 2007 (12), en lo referente a las buenas prácticas de transporte. Así mismo, al ingreso de los vehículos a las plantas se hizo una evaluación visual de sus características estructurales.

Análisis Estadístico

Se realizó el análisis descriptivo de las variables con el programa WIN EPISCOPE 2.0.

\section{RESULTADOS}

El transporte de bovinos en las plantas de beneficio incluidas en el estudio, es realizado en mayor proporción (69\%) por personas con edades comprendidas entre los 31 y 60 años, con amplia experiencia en la conducción; sin embargo, el $92,8 \%$ de éstos, no ha recibido capacitación específica en el transporte de 
animales (Tabla 1). Así mismo, de acuerdo a los requerimientos sanitarios (12), no vigilan las condiciones físicas de los animales durante el viaje, y los manejan con métodos que no garantizan el BA, como el uso de tábanos o picanas eléctricas, torcido de cola u otros métodos que generan dolor, uso de palos, varetas o lazos (Tabla 1).

Tabla 1. Características demográficas y actitudes de los conductores de bovinos respecto al bienestar de los animales durante el transporte hacia las plantas de beneficio evaluadas, 2010

\begin{tabular}{lcclcc}
\hline \multicolumn{1}{c}{ Variables } & $\mathrm{n}$ & $\%$ & \multicolumn{1}{c}{ Variables } & $\mathrm{n}$ & $\%$ \\
\hline Edad (años) & & & Capacitación específica & & \\
$20-30$ & 37 & 19,1 & $\mathrm{Si}$ & 8 & 4,1 \\
$31-40$ & 59 & 30,4 & $\mathrm{No}$ & 180 & 92,8 \\
$41-50$ & 42 & 21,7 & $\mathrm{NI}$ & 6 & 3,1 \\
$51-60$ & 33 & 17 & & & \\
$\geq 61$ & 14 & 7,2 & Levanta animales caídos & 186 & 95,9 \\
$\mathrm{NI}$ & 9 & 4,6 & $\mathrm{Si}$ & 8 & 4,1 \\
& & & No & & \\
Experiencia en años & & & & & \\
$1-5$ & 32 & 16,5 & Método para levantarlos & & \\
$6-10$ & 36 & 18,6 & & & \\
$11-20$ & 54 & 27,8 & Corriente eléctrica & \\
$21-30$ & 40 & 20,6 & Genera dolor en animal & 45 & 24,8 \\
$31-40$ & 18 & 9,3 & Palos o varetas & 30 & 16,1 \\
$\geq 41$ & 14 & 7,2 & Lazos & 24 & 12,9 \\
\hline
\end{tabular}

Tabla 2. Características estructurales de los vehículos utilizados en el transporte de los bovinos hacia las plantas de beneficio evaluadas, 2010

\begin{tabular}{|c|c|c|c|c|c|}
\hline Variables & $\mathrm{N}$ & $\%$ & Variables & $\mathrm{n}$ & $\%$ \\
\hline Uso exclusivo transporte & & & Piso antideslizante & & \\
\hline bovinos & & & $\mathrm{Si}$ & 150 & 77,3 \\
\hline $\mathrm{Si}$ & 40 & 20,6 & No & 44 & 22,7 \\
\hline No & 154 & 79,4 & & & \\
\hline & & & Material del piso & & \\
\hline Uso de cama & & & Metálico & 111 & 74,5 \\
\hline $\mathrm{Si}$ & 187 & 96,4 & Madera & 33 & 22,4 \\
\hline No & 7 & 3,6 & Otro & 5 & 3,1 \\
\hline Material & & & Sistema de drenaje & & \\
\hline Tamo y heno & 33 & 17,4 & $\mathrm{Si}$ & 0 & \\
\hline Aserrín & 150 & 79,4 & No & 194 & 100 \\
\hline \multirow[t]{3}{*}{ Otro } & 6 & 3,2 & Divisiones internas & & \\
\hline & & & Si & 150 & 77,3 \\
\hline & & & No & 44 & 22,7 \\
\hline \multicolumn{6}{|l|}{ Recicla la cama } \\
\hline $\mathrm{Si}$ & 123 & 63,4 & Material divisiones & & \\
\hline No & 71 & 36,6 & Madera & 131 & 85,6 \\
\hline Usa carpa & & & Metálico & 17 & 14,4 \\
\hline $\mathrm{Si}$ & 174 & 89,7 & Bordes carrocería & & \\
\hline \multirow[t]{2}{*}{ No } & 20 & 10,3 & Agudas & 53 & 27,3 \\
\hline & & & Romas & 141 & 72,7 \\
\hline
\end{tabular}


Los vehículos utilizados para el transporte de animales son variados, pero en su mayor proporción (95\%), corresponden a camiones de carrocería de estacas construidas en madera. El servicio no es especializado $(79,4 \%$ y la infraestructura de los vehículos cumple parcialmente con los requerimientos sanitarios (Tabla 2).

\section{DISCUSIÓN}

El entrenamiento y la capacitación del recurso humano responsable del manejo de los bovinos durante la crianza, transporte y sacrificio, es tal vez la estrategia más efectiva para garantizar la implementación de prácticas de BA $(3,13)$. En el presente estudio, sólo el 4,1 \% $(n=8)$ de los 194 transportadores encuestados ha recibido capacitación específica sobre el manejo y transporte de animales. Programas desarrollados en Canadá y el Reino Unido, han demostrado su eficiencia, que han incluido además el reconocimiento de incentivos y la certificación de los transportadores de ganado que cumplan con los lineamientos previstos por las autoridades y los gremios productivos $(3,5)$. Varios estudios han demostrado que la experiencia y la habilidad de los conductores reducen las lesiones del ganado durante el transporte (14).

Si bien la mayoría de los conductores $(95,9 \%)$ procura levantar los animales postrados o caídos durante el viaje, los métodos para hacerlo afecta el BA (Tabla 1). El uso generalizado del tábano o picana eléctrica (46,8\%)puede generar estrés y dolor en los bovinos, así como lesiones en las masas musculares y pérdida de calidad de la canal, aspectos que producen pérdidas económicas por decomisos y disminución del valor comercial de la canal, en la planta de beneficio $(4,6)$. Esta práctica está limitada para ser usada en los casos en que los animales rehúsen a moverse y sólo cuando el bovino disponga de un espacio despejado para avanzar, una vez que se halla descartado una lesión o enfermedad (11). El uso generalizado de elementos eléctricos para la movilización del ganado se presenta de igual forma en España, y se sugiere que puede ser probablemente el resultado de las malas instalaciones para el cargue y descargue, y el desconocimiento de los manejadores sobre prácticas de BA (4).

La normatividad colombiana vigente ha establecido requerimientos relacionados con las condiciones de transporte, requisitos para los transportadores, densidad de carga y requisitos para los vehículos (12). Con relación a este último ítem, los vehículos evaluados cumplen en un nivel aceptable lo relacionado con el uso de carpa, pisos antideslizantes, material 
sanitario de diseño, y divisiones internas para separar los animales (Tabla 2). Sin embargo, los bordes de las carrocerías son agudos (27,3\%), aspecto que puede afectar la integridad física de los bovinos $(5,13)$. La totalidad de los vehículos no contaba con suministro de agua, favoreciendo la deshidratación de los bovinos, teniendo en cuenta que durante el transporte pierden agua a través de la respiración, micción, heces y evaporación por termorregulación $(13,14)$. Adicionalmente, desde el punto de vista sanitario no cuentan con sistemas de drenaje que eviten la fuga de excrementos y orina a la vía (100\%); y es habitual el uso de cama de tamo, heno o aserrín, así como el reciclaje de la misma en el $62 \%$ de los encuestados, proceso que se puede repetir hasta por seis veces, lo cual puede favorecer la transmisión de enfermedades durante el viaje $(11,15)$.

La falta de especialización del transporte terrestre de bovinos se evidenció en el presente trabajo (Tabla 2). Los vehículos son utilizados exclusivamente para el transporte de animales sólo en el 20,6 \% (40/194) de los encuestados. En Colombia no existe una política clara sobre la implementación del BA en la cadena cárnica bovina. Experiencias realizadas por la academia, la industria y el Estado, en Canadá, Estados Unidos, Australia, Chile y Uruguay, demuestran que es posible transferir conocimiento generado por los investigadores en el ámbito del BA e implementarlo en la industria cárnica, lo cual ha generado un posicionamiento de sus productos en el mercado, un valor agregado, y el desarrollo de programas de entrenamiento y capacitación, que han permitido que el sector se fortalezca y mejore su productividad $(1,3,16)$.

Se concluye que en el área de estudio, se requiere la adopción de infraestructura que permita un óptimo manejo de los animales durante el viaje, así como la capacitación de los transportadores, el fomento de prácticas de $\mathrm{BA}$ en todos los usuarios de la cadena cárnica bovina y el desarrollo de investigaciones que evalúen el impacto del BA en la calidad e inocuidad de la carne, así como las pérdidas económicas por la falta de su implementación •

Agradecimientos: Los autores quieren expresar sus agradecimientos a la Vicerrectoría de Investigaciones de la Universidad de Caldas y a COLCIENCIAS (Proyecto Código 1127-489-25244) por el financiamiento de esta investigación. 


\section{REFERENCIAS}

1. Gallo C. Using scientific evidence to inform public policy on the long distance transportation of animals in South America. Vet Ital. 2008; 44(1):113-120.

2. Minka NS, Ayo JO. Effects of loading behaviour and road transport stress on traumatic injuries in cattle transported by road during the hot-dry season. Livestock Sci. 2007; 107:91-95.

3. Schwartzkopf-Genswein KS, Haley DB, Church S, Woods J, O'byrne T. An education and training programme for livestock transporters in Canada. Vetltal. 2008; 44(1):273283.

4. Villarroel M, María GA, Sierra I, Sañudo C, García-Belenguer S, Gebresenbet G. Critical points in the transport of cattle to slaughter in Spain that may compromise the animal's welfare. Vet Rec. 2001; 149:173-176.

5. Mench JA. Farm animal welfare in the USA: farming practices, research, education, regulation, and assurance programs. Appl Anim Behav Sci. 2008; 113: 298-312.

6. Gallo C, Lizondo G, Knowles G. Effects of journey and large time on steers transported to slaughter in Chile. Vet Rec. 2003; 152: 361-364.

7. Mather AE, Innocent GT, McEwen SA, Reilly WJ, Taylor DJ, Steele WB, et al. Risk factors for hide contamination of Scottish cattle at slaughter with Escherichia coli O157. Prevent Vet Med. 2007; 80: 257-270.

8. Cummins E, Nally P, Butler F, Duffy G, O’Brien S. Development and validation of a probabilistic second-order exposure assessment model for Escherichia coli O157:H7 contamination of beef trimmings from Irish meat plants. Meat Sci. 2008; 79: 139-154.

9. Ferguson DM, Warner RD. Have we underestimated the impact of pre-slaughter on meat quality in ruminants? Meat Sci. 2008; 80:12-19.

10. King DA, Schuehle CE, Randel RD, Welsh TH, Oliphint RA, Baird BE, Curley KO, Vann RC, Hale DS, Savell JW. Influence of animal temperament and stress responsiveness on the carcass quality and beef tenderness of feedlot cattle. Meat Sci. 2006; 74:546-556.

11. Organización Mundial de Sanidad Animal. Código Sanitario para los animales terrestres 2010. Título 7 Bienestar de los animales [Internet]. Disponible en: http://oie.int/esp/ normes/mcode/E_summry.htm. Consultado: Agosto 2010.

12. Instituto Colombiano Agropecuario ICA. Resolución 002341 de agosto 23 de 2007. [Internet]. Disponible en: http://www.ica.gov.co/getattachment/0b5de556-cb4a-43a8-a27acd9a2064b1ab/2341.aspx. Consultado:Junio 2010.

13. Gavinelli A, Ferrara M, Simonin D. Formulating policies for the welfare of animals during long distance transportation. Vet Ital. 2008; 44(1):71-86.

14. Cockram MS. Criteria and potential reasons for maximum journey times for farm animals destined for slaughter. Appl Anim Behav Sci.2007; 106:234-243.

15. Bromm DM. The effects of land transport on animal welfare. Rev sci tech Off Int Epiz. 2005; 24(2): 683-691.

16. Grandin T. Transferring results of behavioral research to industry to improve animal welfare on the farm, ranch and the slaughter plant. Appl Anim Behav Sci. 2003; 81: 215-228. 